\title{
New Middle Jurassic kempynin osmylid lacewings from China
}

Yongjie Wang, Zhiqi Liu, Dong Ren, and Chungkun Shih

Acta Palaeontologica Polonica 56 (4), 2011: 865-869 doi: http://dx.doi.org/10.4202/app.2010.0050

Kempyninae are a subfamily of osmylid lacewings whose extant representatives are restricted to the austral continents. Three new osmylids, Jurakempynus sinensis gen. et sp. nov., J. bellatulus gen. et sp. nov., and J. epunctatus gen. et sp. nov. are described from the Middle Jurassic Jiulongshan Formation of Daohugou Village, Inner Mongolia, China.

The new genus Jurakempynus is assigned to the subfamily Kempyninae constituting its oldest known occurrence. It is allied to the Australian genus Kempynus, sharing two synapomorphies: extremely expanded MP space and distally forked

MP2 in the hindwing. The occurrence of Jurakempynus gen. nov. in China (Northern Hemisphere) implies a much wider

geographic range of the Mesozoic osmylid lacewings in comparison to their modern counterparts.

Yongjie Wang [wangyjosmy@yahoo.cn] and Zhiqi Liu [zhiqiliu@cau.edu.cn],

Department of Entomology, China Agricultural University, 2

Yuanmingyuanxilu, Beijing 100193, China; Dong Ren [rendong@ mail.cnu.edu.cn] (corresponding author), College of Life Sciences, Capital Normal University, 105 Xisanhuanbeilu, Beijing 100048, China.

This is an open-access article distributed under the terms of the Creative Commons Attribution License (for details please see creativecommons.org), which permits unrestricted use, distribution, and reproduction in any medium, provided the original author and source are credited. 